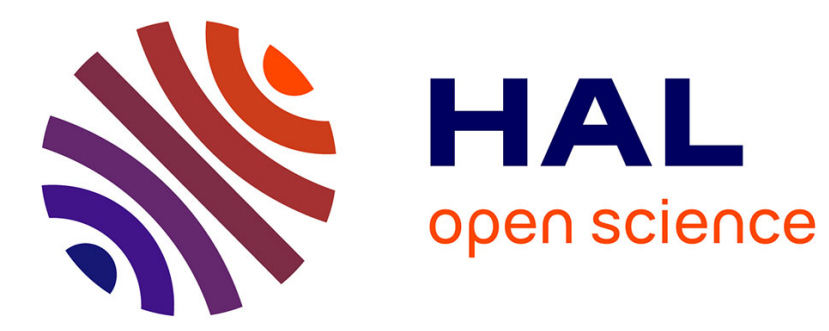

\title{
When Nanoworlds Collide: Implementing DNA Amplification, Nanoparticles, Molecules, and FRET into a Single MicroRNA Biosensor
}

Jingyue Xu, Xue Qiu, Niko Hildebrandt

\section{- To cite this version:}

Jingyue Xu, Xue Qiu, Niko Hildebrandt. When Nanoworlds Collide: Implementing DNA Amplification, Nanoparticles, Molecules, and FRET into a Single MicroRNA Biosensor. Nano Letters, 2021, 21 (11), pp.4802-4808. 10.1021/acs.nanolett.1c01351 . hal-03239156

HAL Id: hal-03239156

https://hal-normandie-univ.archives-ouvertes.fr/hal-03239156

Submitted on 25 Jan 2022

HAL is a multi-disciplinary open access archive for the deposit and dissemination of scientific research documents, whether they are published or not. The documents may come from teaching and research institutions in France or abroad, or from public or private research centers.
L'archive ouverte pluridisciplinaire HAL, est destinée au dépôt et à la diffusion de documents scientifiques de niveau recherche, publiés ou non, émanant des établissements d'enseignement et de recherche français ou étrangers, des laboratoires publics ou privés. 


\section{When Nanoworlds Collide: Implementing DNA Amplification, Nanoparticles, Molecules, and FRET into a Single MicroRNA Biosensor}

Jingyue Xu, ${ }^{1,2}$ Xue Qiu, ${ }^{3,4}$ and Niko Hildebrandt ${ }^{1,2 *}$

1 nanofret.com, Laboratoire Chimie Organique, Bioorganique, Réactivité et Analyse (COBRA), Université de Rouen Normandie, CNRS, INSA, 76821 Mont-Saint-Aignan Cedex, France

2 Institute for Integrative Biology of the Cell (I2BC), Université Paris-Saclay, CNRS, CEA, 91405 Orsay Cedex, France

${ }^{3}$ School of Medicine and Pharmacy, Ocean University of China, 266003 Qingdao, China

${ }^{4}$ Laboratory for Marine Drugs and Bioproducts of Qingdao National Laboratory for Marine Science and Technology, 266237 Qingdao, China

*Corresponding author: niko.hildebrandt@univ-rouen.fr, www.nanofret.com

ABSTRACT: Isothermal nucleic acid amplification strategies have been combined with nanotechnology for advanced biosensing, material design, and biomedical applications. However, merging phenomena and materials of different nanoscales with the aim of exploiting all their benefits at once has remained a challenging endeavor. Here, we exemplify the various problems one can encounter when combining the nanodimensions of lanthanide complexes ( $\sim 2 \mathrm{~nm})$, FRET (Förster resonance energy transfer, $\sim 5 \mathrm{~nm}$ ), QDs (quantum dots, $\sim 20 \mathrm{~nm}$ ), and RCA (rolling circle amplification, $\sim 250 \mathrm{~nm}$ ) into a single microRNA biosensor and how these challenges can be overcome. Six different approaches, including simple FRET-RCA, enzyme-digesting FRET-RCA, and FRET-hyperbranched-RCA were investigated. We demonstrated specific miR-21 detection with $80 \mathrm{fM}$ limit of detection and multiplexing capability with FRET from a Tb complex to different QDs. The detailed view on the various complex multi-nanodimensional assay systems elucidated the limited clinical translation of such sophisticated multicomponent nanobiosensors.

Keywords: quantum dots, terbium, miRNA, rolling circle amplification, diagnostics 
This is the initially submitted version of the paper "J. Xu, X. Qiu, and N. Hildebrandt. When Nanoworlds Collide: Implementing DNA Amplification, Nanoparticles, Molecules, and FRET into a Single MicroRNA Biosensor. Nano Letters 2021, 21(11), 4802-4808."

https://doi.org/10.1021/acs.nanolett.1c01351.

Over the last twenty years, nanotechnology has strongly influenced chemical and biological sensing. For example, the first Letter in the first issue of Nano Letters (2001) reported about colloidal quantum dots (QDs), ${ }^{1}$ which later significantly advanced the fields of fluorescence biosensing and imaging. ${ }^{2-10}$ The cover image of the first issue of ACS Nano (2007) showed DNA molecules packed into nanoengineered polymer microcapsules and nucleic acid nanotechnology, including the design and application of nucleic acid hybrid nanomaterials, has strongly contributed to many research fields, including sensing, imaging, drug delivery, biomedicine, photonics, electronics, biotechnology, and synthetic biology. ${ }^{11-19}$ The first sentence of that issue suggested that "the nanoscale is where disciplines merge and where they bifurcate" 20 and the first article discussed photoinduced electron transfer. ${ }^{21}$ Energy transfer, and in particular FRET (Förster resonance energy transfer), has allowed to scrutinize biomolecular interactions from single molecule pairs to the ensemble level using both spectroscopy and microscopy. ${ }^{22-29}$ Moreover, the combination of FRET and QDs has added significant versatility and performance improvement to photoluminescence (PL) biosensing. ${ }^{30,31}$ These examples illustrate that nucleic acids, energy transfer, QDs, and the merge of their respective research disciplines have been important contributors to science and technology, and their association within a single biosensor appears to be an interesting idea.

Motivated by the many possibilities of combining nucleic acid amplification and nanoparticles for biosensing ${ }^{32-37}$ and our previous results concerning multiplexed detection of microRNAs (miRNAs) via Tb-to-QD FRET ${ }^{38}$ and quantification of endogenous miRNAs from clinical samples via a combination of Tb-to-dye FRET and rolling circle amplification (RCA), ${ }^{39}$ we set forth to develop Tb-to-QD-FRET-RCA for multiplexed miRNA diagnostics. While we could perform a washing-free assay to quantify miR-21 with sub-picomolar limits of detection (LODs), the most important result of our study was the long and rocky road to success, which very well exemplified the reasons that have so far limited the translation of photoluminescent nanoparticles into clinical diagnostics and widely-used sensing and imaging applications. Many studies claim superior performances that outcompete conventional assays and biosensors. However, these new developments cannot be found in many applications afterwards, most probably because the various complications of the experimental design were not sufficiently discussed. Considering the often very complex interactions of nanomaterials and biological molecules and interactions in physiological environments on the nanoscale, it is almost unfair to let potential users discover all 
problems and pitfalls in follow-up studies. In our case, i) FRET occurs over donor-acceptor distances of circa 1 to $10 \mathrm{~nm}$, ii) molecular donors or acceptors display sizes of a few $\mathrm{nm}$, iii) biocompatible QDs have diameters in the range of approximately 5 to $30 \mathrm{~nm}$, and iv) RCA results in coiled DNA structures of several hundreds of nm. ${ }^{40-42}$ Thus, it is arguable if and how those different nanointeractions, nanomaterials, and nanoprocesses can be efficiently merged to simultaneously benefit from all their properties (as represented by the "QD-FRET-DNA flycatliphant" in the abstract image). Here, we will discuss the different approaches (Figure 1), problems, and main results of our study and detail all experimental information and outcomes for each individual step in the comprehensive Supporting Information, such that the results can be fully appreciated and reproduced for further developments in amplified QD-based FRET for advanced biosensing.
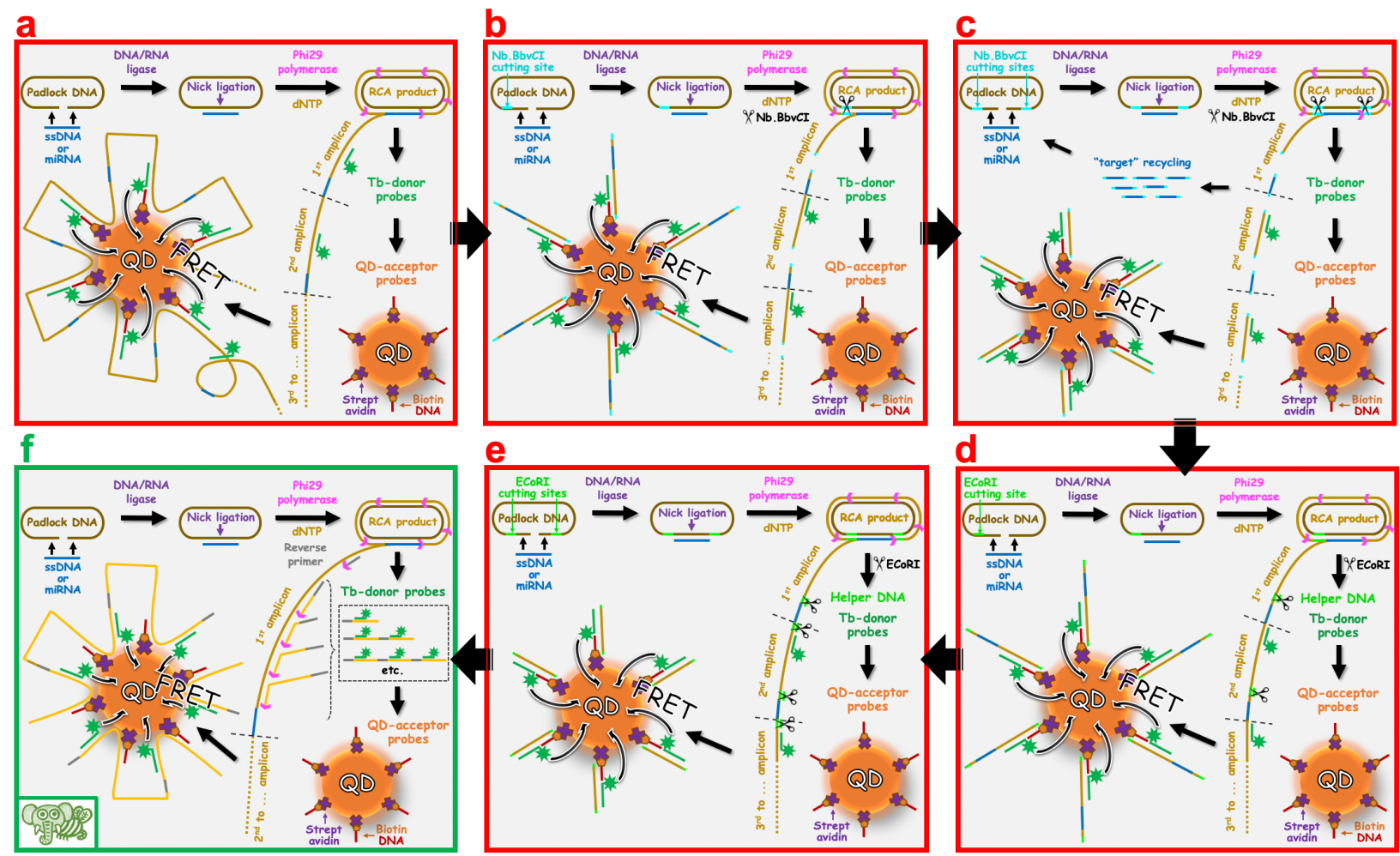

Figure 1: Schematic presentations of different RCA-based approaches toward sensitive and specific miRNA quantification using Tb-to-QD FRET. While all approaches rely on initial RCA (RCA products are shown for only one or two rounds of RCA with each repetitive amplicon separated by thin dashed lines), the strategies toward the final DNA products that combine Tb and QD for FRET were modified for each approach. a: Simple hybridization of Tb and QD FRET probes in the long concatemer RCA product was functional but not very sensitive. $\mathbf{b}$ to e: Different strategies of cutting the RCA product by enzymatic digestion and assembling the Tb-QD FRET pairs by those shorter strands did not improve the assay performance. f: Hyperbranched RCA (HRCA) finally resulted in specific quantification of miR-21 at low pM concentrations and LOD of $\sim 80 \mathrm{fM}$. 
This is the initially submitted version of the paper "J. Xu, X. Qiu, and N. Hildebrandt. When Nanoworlds Collide: Implementing DNA Amplification, Nanoparticles, Molecules, and FRET into a Single MicroRNA Biosensor. Nano Letters 2021, 21(11), 4802-4808."

https://doi.org/10.1021/acs.nanolett.1c01351.

The first attempt of Tb-QD-FRET-RCA (Figure 1a and Figure S2) was inspired by our previous work concerning miRNA and single-stranded (ss)DNA quantification with RCA and Tbto-dye FRET, which resulted in efficient multiplexing, LODs down to $300 \mathrm{aM}$, and quantification of endogenous miRNAs from clinical plasma, cell, and tissue samples. ${ }^{39,43}$ Those studies showed that RCA is a very robust linear amplification approach, which can accomplish more than 1000fold amplification within approximately two hours. We preferred RCA over exponential amplification techniques, which can provide better amplification and lower LODs but are also more complicated and less accurate for sensing. ${ }^{44}$ Tb-based FRET assays can take advantage of the long PL lifetimes of the $\mathrm{Tb}$ donors for time-gated ratiometric FRET detection (ratio of donor and acceptor PL intensities) in a specific time window after pulsed excitation of the donor, which allows for efficient background reduction and washing-free assay formats. On the other hand, Tb-to-dye FRET is significantly different from Tb-to-QD FRET because molecular dye acceptors are much smaller than QD acceptors. Thus, the Tb-to-dye distance is shorter (i.e., higher FRET efficiency) and the hybridization of dye-DNA probes to RCA products (RCPs) is simpler, which means that replacement of dyes by QDs may not be a simple task. Initial results demonstrated hybridization of QD-DNA probes to RCPs that were formed on microscope slides upon DNA target recognition (Figure S3), and a FRET assay with both $\mathrm{Tb}$ and QD DNA probes could quantify sub-pM concentrations of miRNA (Figure S4) when Tb-DNA and biotin-DNA (both of them small) were pre-hybridized to the RCPs and QD-streptavidin was added afterwards. However, the next step to accomplish multiplexing (one major advantage of QDs), in which QD-DNA conjugates were preassembled (DNA-biotin + streptavidin-QDs) did not result in measurable FRET at sub-pM miRNA concentrations (Figure S5). Thus, in contrast to smaller DNA conjugates, the QD-DNA probes could not efficiently hybridize to the RCPs, indicating that an approximately 1000-fold amplified RCA concatemer is too densely coiled within its overall size of a few hundred nm and provides limited accessibility for relatively large nanoparticle-DNA conjugates.

The logical consequence of a too large and too dense RCP would be to reduce it to smaller pieces, e.g., by using endonucleases. Thus, we designed a padlock probe with a specific sequence that produced a restriction site in the newly synthesized padlock-complementary DNA strand (Figure 1b and Figure S6). A nicking endonuclease ( $N b . B b v C I)$ would then hydrolyze only the restriction site (GGAGTCG between $\mathrm{T}$ and $\mathrm{C}$ ) of the amplified ssDNA strand on the padlock-DNA and leave the padlock itself intact. This theoretically ideal approach, inspired by studies that used 
This is the initially submitted version of the paper "J. Xu, X. Qiu, and N. Hildebrandt. When Nanoworlds Collide: Implementing DNA Amplification, Nanoparticles, Molecules, and FRET into a Single MicroRNA Biosensor. Nano Letters 2021, 21(11), 4802-4808."

https://doi.org/10.1021/acs.nanolett.1c01351.

miRNA-induced RCA and subsequent cutting via $\mathrm{Nb} . \mathrm{BbvCI},{ }^{45,46}$ did unfortunately not translate into practice because there was no difference in performance compared to the first assay both with and without addition of the enzyme (Figure S7). Addition of a second restriction site to improve the cutting performance and to recycle the target sequence for additional amplification (Figure 1c and Figure S8) looked like an intriguing idea but only further reduced the assay performance (Figure S9). Because one restriction site in the amplified DNA did not influence the assay performance and a second one even reduced it, we concluded that simultaneous RCA and RCP digestion into smaller ssDNA strands is not efficient. There is probably a competition between the phi29 polymerase amplification reaction (synthesizing new DNA around the circularized padlock) and the endonuclease cutting reaction (cutting of the RCP on the padlock), such that the combined reactions do not function as efficiently as if they were separated. Thus, we would need to sacrifice assay simplicity (single-step amplification and cutting) by separating RCA and enzymatic digestion in two separate steps.

To realize such a separated amplification/digestion approach, we designed a new concept (Figure 1d and Figure S10), in which the padlock probe induced a restriction site for EcoRI endonuclease that specifically cuts double-stranded (ds)DNA between G and A in the G/AATTC and complementary CTTAA/G sequences. After completed RCA, EcoRI (with the help of a GAATTC "helper" DNA that hybridizes to the RCP) cut the RCP in specific short ssDNA sequences that could be used for initiating Tb-to-QD FRET. We first confirmed that the short ssDNA sequences (cut RCP) can be applied for multiplexed Tb-to-QD FRET sensing using three different streptavidin-coated QDs (emitting at 605, 655, and $705 \mathrm{~nm}$, respectively), biotin-DNA, and Tb-DNA (Figure S11). Then, we confirmed the functionality of the padlock for target-primed RCA FRET detection (Figure S12) and the EcoRI cutting efficiency at different reaction times and concentrations (Figure S13). Despite those verifications of efficient Tb-to-QD FRET, RCA, and EcoRI-dependent dsDNA digestion, the final assay was not functional (Figure S14). Thus, we decided to run various control and optimization experiments to better understand the reasons for failure. Further variation of the EcoRI concentration did not improve the assay and even Tb-to-dye FRET (without QDs) using the same RCP-cutting principle (to rule out that the QD size was the problem) did not result in significant FRET signals (Figure S15). Hybridizing biotin-DNA first and binding streptavidin-QD afterwards, which was functional in the simple RCA FRET assay, failed (Figure S16). Using miRNA instead of ssDNA targets (to be sure that the target was not the 
This is the initially submitted version of the paper "J. Xu, X. Qiu, and N. Hildebrandt. When Nanoworlds Collide: Implementing DNA Amplification, Nanoparticles, Molecules, and FRET into a Single MicroRNA Biosensor. Nano Letters 2021, 21(11), 4802-4808."

https://doi.org/10.1021/acs.nanolett.1c01351.

issue) and increasing the target concentrations resulted in weak signals only for Tb-to-dye but not for Tb-to-QD FRET (Figure S17). A strictly controlled temperature program (to expand the coiled RCP and improve the accessibility of the QD-DNA for RCP hybridization) yielded only minor assay improvements, still far below the standard (Tb-to-dye) RCA FRET performance (Figure S18). Complete miR-21 assays under different EcoRI reaction times and concentrations did not lead to significant performance enhancement (Figures S19 and S20). Changing the buffer conditions (to improve enzymatic digestion) and deactivating EcoRI after the cutting reaction (to avoid interference with RCP-probe hybridization) did not change the assay functionality (Figure S21). Increasing the helper DNA concentration (to make sure that the restriction site is dsDNA) and the biotin-DNA per streptavidin-QD ratio (to provide sufficient amount of probe DNA for RCP hybridization) failed (Figures S22 and S23). Because all possible optimizations did not improve the FRET signals and the assay sensitivity was independent of EcoRI (Figure S24), we concluded that the enzymatic digestion of the RCP within the complete assay was not efficient enough to provide the necessary $\mathrm{nM}$ concentrations of short ssDNA strands for hybridization with the $\mathrm{Tb}$ and QD DNA probes and efficient FRET (as demonstrated in Figure S11). Exchanging QD605 with QD705 (to obtain better Tb-to-QD FRET performance) resulted in significant FRET but only for miRNAs at pM concentrations (Figure S25) and was much less efficient compared to the initial hybridization experiments (Figure S11c) or the Tb-to-dye RCA FRET “gold standard". Even assays at concentrations that were similar to polyacrylamide gel electrophoresis (PAGE) conditions (10-fold higher padlock concentration and 1000-fold higher miR-21 concentrations), for which a cutting of the RCP in smaller sequences was clearly demonstrated (Figure S26), did only result in significant FRET (but still low for the very high target and padlock concentrations) when no EcoRI was present (Figure S27a), which showed that RCA was functional but enzymatic digestion of the RCP did not yield the adequate short ssDNA strands for Tb and QD DNA probe hybridization. Thus, we decided to improve the cutting efficiency by adding a second EcoRI-specific restriction site (Figure 1e and Figure S28). This approach slightly improved the assay sensitivity even for Tb-to-QD605 FRET (Figure S29) but still with only very weak FRET signals that were approximately 30-fold lower compared to conventional Tb-to-dye RCA FRET with the same padlock probe but without RCP digestion (Figure S30). Despite the demonstrated principle of cutting the RCP in smaller pieces, such that they would fit into our previous Tb-to-QD FRET detection scheme, the results of the many control experiments led to the conclusion that the overall 
This is the initially submitted version of the paper "J. Xu, X. Qiu, and N. Hildebrandt. When Nanoworlds Collide: Implementing DNA Amplification, Nanoparticles, Molecules, and FRET into a Single MicroRNA Biosensor. Nano Letters 2021, 21(11), 4802-4808."

https://doi.org/10.1021/acs.nanolett.1c01351.

assay environment (with multiple enzymes, multiple assay components, and multiple steps) was too complex to result in efficient hybridization of the Tb and QD DNA probes with the final reaction product (amplified and cut DNA) and that many more optimization steps will be necessary to actually translate the concept into a sensitive and reproducible miRNA assay.

Inspired by another approach that used QD-based hyperbranched RCA (HRCA) FRET multiplexing, ${ }^{47}$ we wanted to test its applicability to target-primed RCA and Tb-to-QD FRET (Figure 1f and Figure S31). We first verified the capability of the padlock to produce miRNAtarget-specific RCPs and shorter hyperbranched RCPs in the presence of a reverse primer (Figure S32). We also directly used the best-performing Tb-QD705 FRET pair and optimized padlock and reverse primer concentration, DNA-biotin per streptavidin-QD ratio, and HRCA reaction time (Figure S33). The assays showed significant miR-21-specific FRET over a broad dynamic concentration range (between $3 \mathrm{pM}$ and $500 \mathrm{pM}$ - Figure S34) and assay functionality for both Tb-QD705 and Tb-QD650 FRET pairs to demonstrate its multiplexing capability (Figure 2 and Figure S35). Although the FRET-sensitized PL decay curves of both QD650 (Figure 2a) and QD705 (Figure 2c) showed significant miR-21 concentration-dependent intensity increase of the FRET-related PL decays (shorter decay component that is mainly present in the detection window time-range shown in gray in Figure 2 a and c), those FRET-sensitized PL decays are still relatively long, thus, excluding a shorter Tb-QD distance compared to the other approaches. We therefore concluded that both the exponential amplification of HRCA and the higher hybridization efficiencies of hyperbranched RCPs with Tb and QD DNA probes were the main reasons for the significantly better assay performance. Although the spectral overlap of Tb PL and QD absorption is very similar for both Tb-QD FRET pairs (Figure S1a), the better FRET performance of QD705 ( $\sim 5$-fold relative FRET increase in Figure 2d) compared to QD650 ( 1.3-fold increase in Figure 2b) was mainly related to the lower Tb PL background in the QD705 detection channel, which measured PL beyond 700 nm (Figure S1b). 

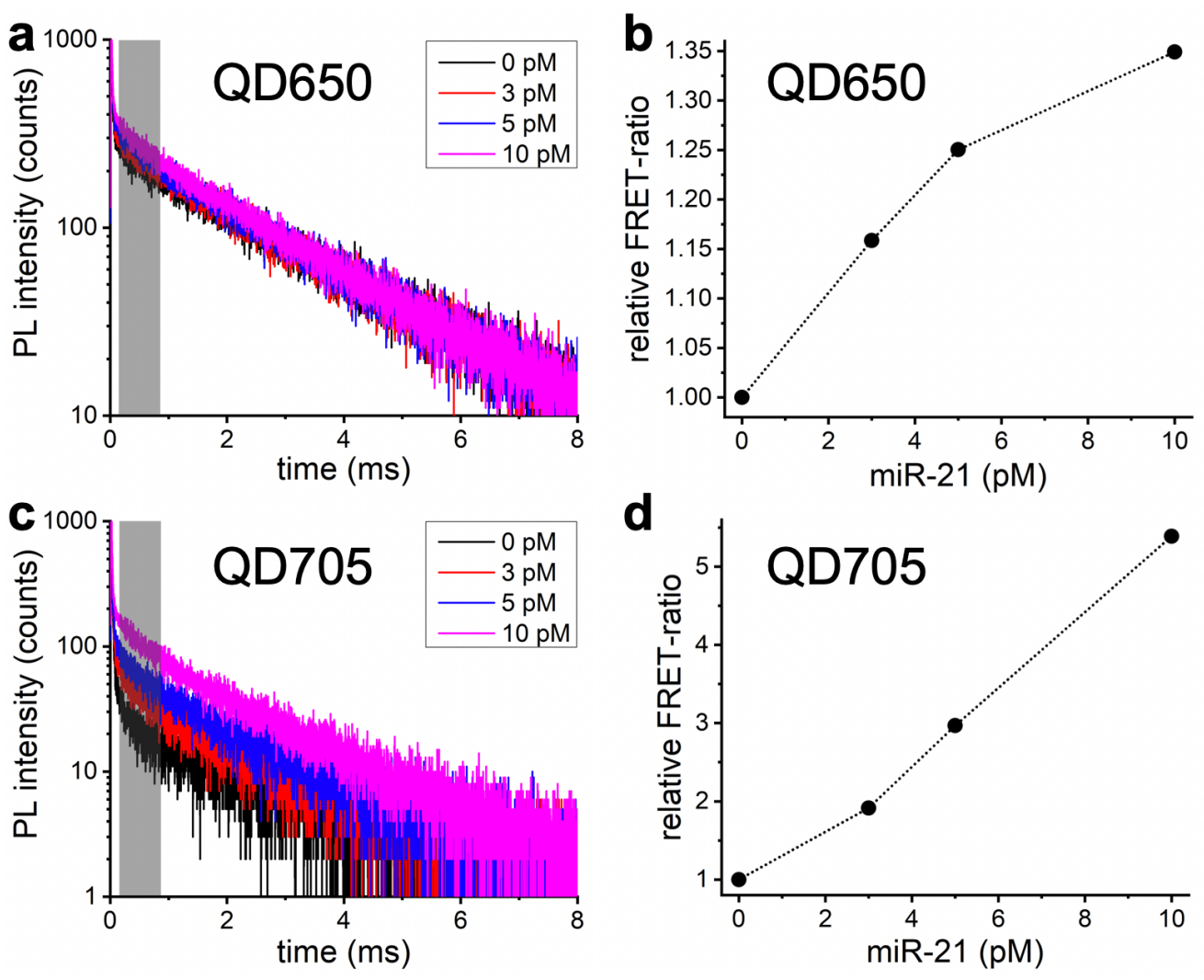

Figure 2: The Tb-QD-FRET-HRCA assay for quantifying low pM concentrations of miR-21 can be applied to different QDs (a,b for QD650 and c,d for QD705), demonstrating its multiplexing capability. a,c: QDacceptor PL decay curves, whose intensities increase with increasing miR-21 target concentration due to FRET sensitization from Tb donors. The absolute intensity increase (not well visible due to the logarithmic intensity scale) is similar for QD650 and QD705 (cf. Figure S35). b,d: The FRET ratios (ratio of time-gated QD and Tb PL intensities between 0.1 and $0.9 \mathrm{~ms}$ after the excitation pulse, i.e., time range shown in gray in $\mathbf{a}$ and $\mathbf{c}$ ) show that the relative FRET increase is much higher for QD705 because of the significantly lower Tb background PL (black curves in a and c) in the QD705 detection channel (cf. Figure S1).

The miR-21 assay was very specific (measured against miR-20b - Figure 3a and Figure S36), as expected from previously demonstrated single-nucleotide variant specificity for the same miR-21 target-primed RCA approach. ${ }^{39}$ The estimated LOD was $80 \mathrm{fM}$ (Figure 3b,c and Figure S37). First attempts to perform actual multiplexing of two different miRNA targets showed that also this functional Tb-QD-FRET-HRCA system is highly complex, and translation to other targets is not as straightforward as for RCA without hyperbranching. Two different padlock and sensor designs for miR-20a resulted in relatively low assay performance and the production of only few hyperbranched RCPs (Figures S38 to S40), whereas the miR-20b (only two mismatched nucleotides compared to miR-20a) resulted in more hyperbranched RCP production and better assay performance, which was still significantly lower compared to the miR-21 HRCA sensor 
This is the initially submitted version of the paper "J. Xu, X. Qiu, and N. Hildebrandt. When Nanoworlds Collide: Implementing DNA Amplification, Nanoparticles, Molecules, and FRET into a Single MicroRNA Biosensor. Nano Letters 2021, 21(11), 4802-4808."

https://doi.org/10.1021/acs.nanolett.1c01351.

(Figures S41 and S42). Considering that the padlock design influences both RCA and hyperbranching amplification, it is reasonable that a combined padlock and reverse primer optimization for each specific miRNA target is more complicated than for simple RCA. However, the fully functional proof of concept demonstrated that there is no fundamental limitation and that multiplexed miRNA detection from a single sample will become possible upon further systematic optimization.
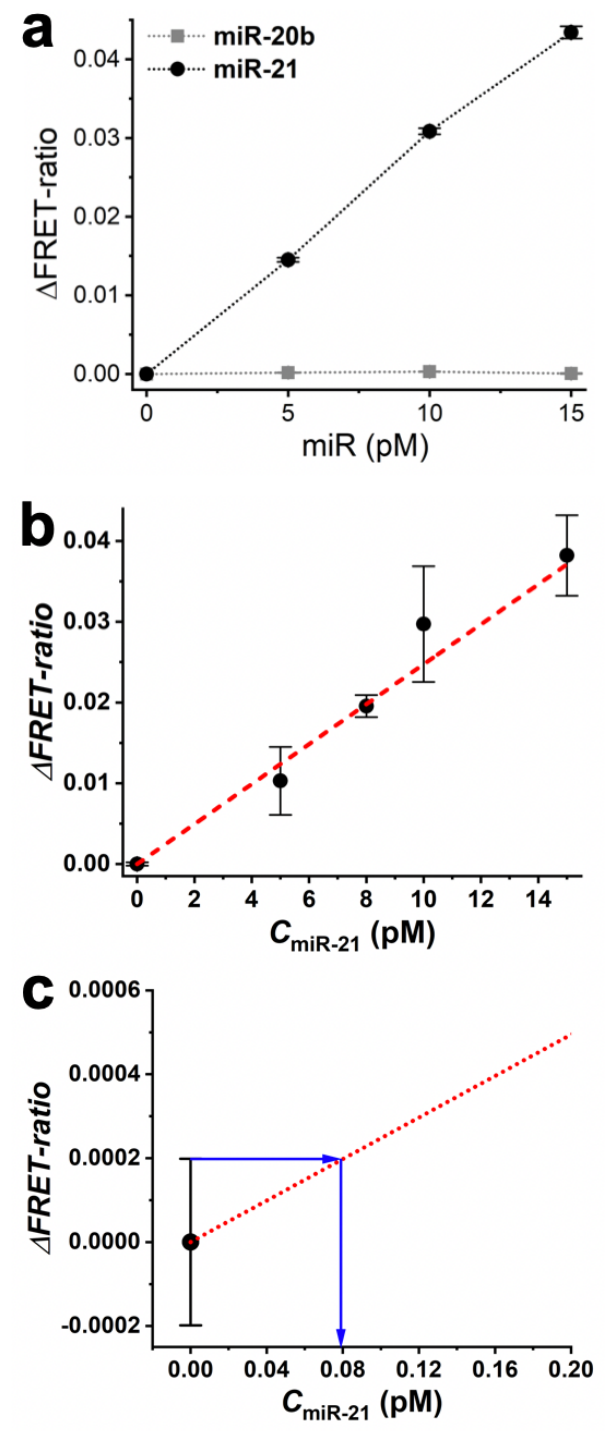

Figure 3: The Tb-QD705-FRET-HRCA assay showed excellent specificity tested against miR-20b (a) and strong miR-21 concentration-dependent FRET-ratio increase at low pM concentrations (b). A LOD of $80 \mathrm{fM}$ was estimated as the concentration corresponding to the $\triangle F R E T$-ratio value 3 standard deviations above the $\triangle$ FRET-ratio without target (c). $\triangle$ FRET-ratio signifies the FRET-ratio at a given concentration minus the FRET-ratio without target (zero concentration). Error bars correspond to the standard deviations from 3 independent measurements $(n=3)$ for all target concentrations except for no target (zero concentration), which used 15 measurements $(n=15)$. 
This is the initially submitted version of the paper "J. Xu, X. Qiu, and N. Hildebrandt. When Nanoworlds Collide: Implementing DNA Amplification, Nanoparticles, Molecules, and FRET into a Single MicroRNA Biosensor. Nano Letters 2021, 21(11), 4802-4808."

https://doi.org/10.1021/acs.nanolett.1c01351.

In summary, our results demonstrate that the design and application of mutli-nano-hybrid biosensors is significantly more complex than most often shown and discussed in the literature, which is a major reasons for limited reproducibility and clinical translation of such assay systems. While we could demonstrate a washing-free and specific miR-21 assay with sub-picomolar LOD and multiplexing capability, the development from initial attempts to the final result required significantly more modification and optimization steps compared to purely molecular systems (without nanoparticles and/or nucleic acid amplification). Our detailed experimental investigation suggested that simple RCA resulted in large $(>100 \mathrm{~nm})$ but too dense (coiled structure) concatemers with limited hybridization efficiency for the relatively large $(>10 \mathrm{~nm})$ QD-DNA probes in parallel to the relatively small $(<5 \mathrm{~nm})$ Tb-DNA probes. Various approaches of cutting the RCA products into smaller DNA strands using specific $\mathrm{Nb.BbvCI}$ (for simultaneous RCA and RCP digestion) or ECoRI (for separated RCA and RCP digestion) endonucleases resulted in too complex amplification systems. The competing enzymatic reactions interfered with an efficient reduction of the RCPs into short ssDNA strands for efficient Tb and QD DNA-probe hybridization and could therefore not improve the assay performance. Using the exponential HRCA approach yielded short enough hyperbranched RCPs that were able to trigger hybridization with both Tb FRET donor and QD FRET acceptor DNA probes and allowed for quantifying miR-21 with an LOD of $\sim 80 \mathrm{fM}$, which is lower than for enzyme-free and washing-free amplified Tb-to-dye FRET assays $(1.7 \mathrm{pM})^{48}$ but higher than for Tb-to-dye RCA FRET assays (30 fM). ${ }^{39}$ Considering that QD-to-dye HRCA assays without target primed RCA (circular DNA templates with limited target specificity) could result in sub-fM LODs, ${ }^{47}$ our proof-of-concept multi-nano-dimensional Tb-to-QD FRET sensor demonstrated that further optimization can potentially result in sensitive, specific, and multiplexed miRNA quantification. We believe that our results present an important guideline to the nanobiosensing community for the challenging endeavor of translating the photophysical and dimensional advantages of nanotechnology into advanced nucleic acid clinical diagnostics and research. 
This is the initially submitted version of the paper "J. Xu, X. Qiu, and N. Hildebrandt. When Nanoworlds Collide: Implementing DNA Amplification, Nanoparticles, Molecules, and FRET into a Single MicroRNA Biosensor. Nano Letters 2021, 21(11), 4802-4808."

https://doi.org/10.1021/acs.nanolett.1c01351.

\section{ASSOCIATED CONTENT \\ Supporting Information}

All experimental details and results (6 Tables and 42 Figures) from 34 different assays performed to investigate the nanobiosensor development.

\section{ACKNOWLEDGEMENTS}

We thank Lumiphore, Inc. for the gift of Lumi4 reagents. This work was supported by the French National Research Agency (ANR projects "neutrinos", "PhenX", and Labex "SynOrg”), the French "Institut National du Cancer" and "Direction Générale de l'Offre de Soins" (INCa and DGOS; project PRTk 16158 - Gynomir), the Institut Universitaire de France (IUF), and the China Scholarship Council (CSC).

\section{REFERENCES}

(1) Norris, D. J.; Yao, N.; Charnock, F. T.; Kennedy, T. A. High-Quality Manganese-Doped ZnSe Nanocrystals. Nano Lett. 2001, 1 (1), 3-7. https://doi.org/10.1021/nl005503h.

(2) Michalet, X.; Pinaud, F. F.; Bentolila, L. A.; Tsay, J. M.; Doose, S.; Li, J. J.; Sundaresan, G.; Wu, A. M.; Gambhir, S. S.; Weiss, S. Quantum Dots for Live Cells, in Vivo Imaging, and Diagnostics. Science 2005, 307 (5709), 538-544. https://doi.org/10.1126/science.1104274.

(3) Zrazhevskiy, P.; Sena, M.; Gao, X. Designing Multifunctional Quantum Dots for Bioimaging, Detection, and Drug Delivery. Chem. Soc. Rev. 2010, 39 (11), 4326-4354. https://doi.org/10.1039/b915139g.

(4) Freeman, R.; Willner, I. Optical Molecular Sensing with Semiconductor Quantum Dots (QDs). Chem. Soc. Rev. 2012, 41 (10), 4067-4085. https://doi.org/10.1039/c2cs15357b.

(5) Kovalenko, M. V.; Manna, L.; Cabot, A.; Hens, Z.; Talapin, D. V.; Kagan, C. R.; Klimov, V. I.; Rogach, A. L.; Reiss, P.; Milliron, D. J.; Guyot-Sionnnest, P.; Konstantatos, G.; Parak, W. J.; Hyeon, T.; Korgel, B. A.; Murray, C. B.; Heiss, W. Prospects of Nanoscience with Nanocrystals. ACS Nano 2015, 9 (2), $1012-1057$. https://doi.org/10.1021/nn506223h.

(6) Zhou, J.; Yang, Y.; Zhang, C. Toward Biocompatible Semiconductor Quantum Dots: From Biosynthesis and Bioconjugation to Biomedical Application. Chem. Rev. 2015, 115 (21), 11669-11717. https://doi.org/10.1021/acs.chemrev.5b00049.

(7) Xu, G.; Zeng, S.; Zhang, B.; Swihart, M. T.; Yong, K.-T.; Prasad, P. N. New Generation Cadmium-Free Quantum Dots for Biophotonics and Nanomedicine. Chem. Rev. 2016, 116 (19), 12234-12327. https://doi.org/10.1021/acs.chemrev.6b00290.

(8) Wu, P.; Hou, X.; Xu, J.-J.; Chen, H.-Y. Ratiometric Fluorescence, Electrochemiluminescence, and Photoelectrochemical Chemo/Biosensing Based on Semiconductor Quantum Dots. Nanoscale 2016, 8 (16), 8427-8442. https://doi.org/10.1039/c6nr01912a.

(9) Owens, J.; Brus, L. Chemical Synthesis and Luminescence Applications of Colloidal Semiconductor Quantum Dots. J. Am. Chem. Soc. 2017, 139 (32), 10939-10943. https://doi.org/10.1021/jacs.7b05267.

(10) Saeboe, A. M.; Nikiforov, A. Yu.; Toufanian, R.; Kays, J. C.; Chern, M.; Casas, J. P.; Han, K.; Piryatinski, A.; Jones, D.; Dennis, A. M. Extending the Near-Infrared Emission Range of Indium Phosphide Quantum Dots for Multiplexed In Vivo Imaging. Nano Lett. 2021, 21 (7), 3271-3279. https://doi.org/10.1021/acs.nanolett.1c00600.

(11) Lu, C.-H.; Willner, B.; Willner, I. DNA Nanotechnology: From Sensing and DNA Machines to Drug-Delivery Systems. ACS Nano 2013, 7 (10), 8320-8332. https://doi.org/10.1021/nn404613v.

(12) Jasinski, D.; Haque, F.; Binzel, D. W.; Guo, P. Advancement of the Emerging Field of RNA Nanotechnology. ACS Nano 2017, 11 (2), 1142-1164. https://doi.org/10.1021/acsnano.6b05737.

(13) Chidchob, P.; Sleiman, H. F. Recent Advances in DNA Nanotechnology. Curr. Opin. Chem. Biol. 2018, 46, 63-70. https://doi.org/10.1016/j.cbpa.2018.04.012. 
This is the initially submitted version of the paper "J. Xu, X. Qiu, and N. Hildebrandt. When Nanoworlds Collide: Implementing DNA Amplification, Nanoparticles, Molecules, and FRET into a Single MicroRNA Biosensor. Nano Letters 2021, 21(11), 4802-4808."

https://doi.org/10.1021/acs.nanolett.1c01351.

(14) Hendrikse, S. I. S.; Gras, S. L.; Ellis, A. V. Opportunities and Challenges in DNA-Hybrid Nanomaterials. ACS Nano 2019, 13 (8), 8512-8516. https://doi.org/10.1021/acsnano.9b06186.

(15) Madsen, M.; Gothelf, K. V. Chemistries for DNA Nanotechnology. Chem. Rev. 2019, 119 (10), 6384-6458. https://doi.org/10.1021/acs.chemrev.8b00570.

(16) Afonin, K. A.; Dobrovolskaia, M. A.; Church, G.; Bathe, M. Opportunities, Barriers, and a Strategy for Overcoming Translational Challenges to Therapeutic Nucleic Acid Nanotechnology. ACS Nano 2020, 14 (8), 9221-9227. https://doi.org/10.1021/acsnano.0c04753.

(17) Kim, J.; Franco, E. RNA Nanotechnology in Synthetic Biology. Curr. Opin. Biotechnol. 2020, 63, 135-141. https://doi.org/10.1016/j.copbio.2019.12.016.

(18) Ebrahimi, S. B.; Samanta, D.; Mirkin, C. A. DNA-Based Nanostructures for Live-Cell Analysis. J. Am. Chem. Soc. 2020, 142 (26), 11343-11356. https://doi.org/10.1021/jacs.0c04978.

(19) DeLuca, M.; Shi, Z.; Castro, C. E.; Arya, G. Dynamic DNA Nanotechnology: Toward Functional Nanoscale Devices. Nanoscale Horiz. 2020, 5 (2), 182-201. https://doi.org/10.1039/c9nh00529c.

(20) Weiss, P. S. Welcome to ACS Nano. ACS Nano 2007, 1 (1), 1-1. https://doi.org/10.1021/nn700131b.

(21) Kongkanand, A.; Kamat, P. V. Electron Storage in Single Wall Carbon Nanotubes. Fermi Level Equilibration in Semiconductor-SWCNT Suspensions. ACS Nano 2007, 1 (1), 13-21. https://doi.org/10.1021/nn700036f.

(22) Molecular Imaging - FRET Microscopy and Spectroscopy; Periasamy, A., Day, R. N., Eds.; Elsevier, 2005. https://doi.org/10.1016/B978-0-19-517720-6.X5006-8.

(23) Medintz, I. L.; Hildebrandt, N. FRET - Förster Resonance Energy Transfer: From Theory to Applications; John Wiley \& Sons, 2013.

(24) Okamoto, K.; Sako, Y. Recent Advances in FRET for the Study of Protein Interactions and Dynamics. Curr. Opin. Struct. Biol. 2017, 46, 16-23.

(25) Bunt, G.; Wouters, F. S. FRET from Single to Multiplexed Signaling Events. Biophys. Rev. 2017, 9 (2), 119129. https://doi.org/10.1007/s12551-017-0252-z.

(26) Teunissen, A. J. P.; Perez-Medina, C.; Meijerink, A.; Mulder, W. J. M. Investigating Supramolecular Systems Using Forster Resonance Energy Transfer. Chem. Soc. Rev. 2018, 47 (18), 7027-7044. https://doi.org/10.1039/c8cs00278a.

(27) Hellenkamp, B.; Schmid, S.; Doroshenko, O.; Opanasyuk, O.; Kühnemuth, R.; Rezaei Adariani, S.; Ambrose, B.; Aznauryan, M.; Barth, A.; Birkedal, V.; Bowen, M. E.; Chen, H.; Cordes, T.; Eilert, T.; Fijen, C.; Gebhardt, C.; Götz, M.; Gouridis, G.; Gratton, E.; Ha, T.; Hao, P.; Hanke, C. A.; Hartmann, A.; Hendrix, J.; Hildebrandt, L. L.; Hirschfeld, V.; Hohlbein, J.; Hua, B.; Hübner, C. G.; Kallis, E.; Kapanidis, A. N.; Kim, J.Y.; Krainer, G.; Lamb, D. C.; Lee, N. K.; Lemke, E. A.; Levesque, B.; Levitus, M.; McCann, J. J.; NarediRainer, N.; Nettels, D.; Ngo, T.; Qiu, R.; Robb, N. C.; Röcker, C.; Sanabria, H.; Schlierf, M.; Schröder, T.; Schuler, B.; Seidel, H.; Streit, L.; Thurn, J.; Tinnefeld, P.; Tyagi, S.; Vandenberk, N.; Vera, A. M.; Weninger, K. R.; Wünsch, B.; Yanez-Orozco, I. S.; Michaelis, J.; Seidel, C. A. M.; Craggs, T. D.; Hugel, T. Precision and Accuracy of Single-Molecule FRET Measurements-a Multi-Laboratory Benchmark Study. Nat. Methods 2018, 15 (9), 669-676. https://doi.org/10.1038/s41592-018-0085-0.

(28) Chen, T.; He, B.; Tao, J.; He, Y.; Deng, H.; Wang, X.; Zheng, Y. Application of Forster Resonance Energy Transfer (FRET) Technique to Elucidate Intracellular and In Vivo Biofate of Nanomedicines. Adv. Drug Deliv. Rev. 2019, 143, 177-205. https://doi.org/10.1016/j.addr.2019.04.009.

(29) Algar, W. R.; Hildebrandt, N.; Vogel, S. S.; Medintz, I. L. FRET as a Biomolecular Research ToolUnderstanding Its Potential While Avoiding Pitfalls. Nat. Methods 2019, 16 (9), 815-829. https://doi.org/10.1038/s41592-019-0530-8.

(30) Hildebrandt, N.; Spillmann, C. M.; Algar, W. R.; Pons, T.; Stewart, M. H.; Oh, E.; Susumu, K.; Diaz, S. A.; Delehanty, J. B.; Medintz, I. L. Energy Transfer with Semiconductor Quantum Dot Bioconjugates: A Versatile Platform for Biosensing, Energy Harvesting, and Other Developing Applications. Chem. Rev. 2017, 117 (2, SI), 536-711. https://doi.org/10.1021/acs.chemrev.6b00030.

(31) Cardoso Dos Santos, M.; Algar, W. R.; Medintz, I. L.; Hildebrandt, N. Quantum Dots for Förster Resonance Energy Transfer (FRET). TrAC Trends Anal. Chem. 2020, 125, 115819. https://doi.org/10.1016/j.trac.2020.115819.

(32) Zhao, W.; Ali, M. M.; Brook, M. A.; Li, Y. Rolling Circle Amplification: Applications in Nanotechnology and Biodetection with Functional Nucleic Acids. Angew. Chem. Int. Ed Engl. 2008, 47 (34), 6330-6337. https://doi.org/10.1002/anie.200705982.

(33) Wu, L.; Xiong, E.; Zhang, X.; Zhang, X.; Chen, J. Nanomaterials as Signal Amplification Elements in DNABased Electrochemical Sensing. Nano Today 2014, 9 (2), 197-211. https://doi.org/10.1016/j.nantod.2014.04.002. 
This is the initially submitted version of the paper "J. Xu, X. Qiu, and N. Hildebrandt. When Nanoworlds Collide: Implementing DNA Amplification, Nanoparticles, Molecules, and FRET into a Single MicroRNA Biosensor. Nano Letters 2021, 21(11), 4802-4808."

https://doi.org/10.1021/acs.nanolett.1c01351.

(34) Wang, L.; Arrabito, G. Hybrid, Multiplexed, Functional DNA Nanotechnology for Bioanalysis. Analyst 2015, 140 (17), 5821-5848. https://doi.org/10.1039/C5AN00861A.

(35) Zhou, H.; Liu, J.; Xu, J.-J.; Zhang, S.-S.; Chen, H.-Y. Optical Nano-Biosensing Interface via Nucleic Acid Amplification Strategy: Construction and Application. Chem. Soc. Rev. 2018, 47 (6), 1996-2019. https://doi.org/10.1039/c7cs00573c.

(36) Chen, Y.-X.; Huang, K.-J.; Niu, K.-X. Recent Advances in Signal Amplification Strategy Based on Oligonucleotide and Nanomaterials for MicroRNA Detection-a Review. Biosens. Bioelectron. 2018, 99, 612624. https://doi.org/10.1016/j.bios.2017.08.036.

(37) Xiao, M.; Lai, W.; Man, T.; Chang, B.; Li, L.; Chandrasekaran, A. R.; Pei, H. Rationally Engineered Nucleic Acid Architectures for Biosensing Applications. Chem. Rev. 2019, 119 (22), 11631-11717. https://doi.org/10.1021/acs.chemrev.9b00121.

(38) Qiu, X.; Hildebrandt, N. Rapid and Multiplexed MicroRNA Diagnostic Assay Using Quantum Dot-Based Förster Resonance Energy Transfer. ACS Nano 2015, 9 (8), 8449-8457. https://doi.org/10.1021/acsnano.5b03364.

(39) Qiu, X.; Xu, J.; Guo, J.; Yahia-Ammar, A.; Kapetanakis, N.-I.; Duroux-Richard, I.; Unterluggauer, J. J.; Golob-Schwarzl, N.; Regeard, C.; Uzan, C.; Gouy, S.; DuBow, M.; Haybaeck, J.; Apparailly, F.; Busson, P.; Hildebrandt, N. Advanced MicroRNA-Based Cancer Diagnostics Using Amplified Time-Gated FRET. Chem. Sci. 2018, 9 (42), 8046-8055. https://doi.org/10.1039/c8sc03121e.

(40) Han, D.; Park, Y.; Nam, H.; Lee, J. B. Enzymatic Size Control of RNA Particles Using Complementary Rolling Circle Transcription (CRCT) Method for Efficient SiRNA Production. Chem. Commun. 2014, 50 (79), 11665-11667. https://doi.org/10.1039/C4CC04969A.

(41) Lee, S. Y.; Kim, K.-R.; Bang, D.; Bae, S. W.; Kim, H. J.; Ahn, D.-R. Biophysical and Chemical Handles to Control the Size of DNA Nanoparticles Produced by Rolling Circle Amplification. Biomater. Sci. 2016, 4 (9), 1314-1317. https://doi.org/10.1039/C6BM00296J.

(42) Yuan, X.; Xiao, F.; Zhao, H.; Huang, Y.; Shao, C.; Weizmann, Y.; Tian, L. High-Yield Method To Fabricate and Functionalize DNA Nanoparticles from the Products of Rolling Circle Amplification. ACS Appl. Bio Mater. 2018, 1 (2), 511-519. https://doi.org/10.1021/acsabm.8b00238.

(43) Qiu, X.; Guo, J.; Xu, J.; Hildebrandt, N. Three-Dimensional FRET Multiplexing for DNA Quantification with Attomolar Detection Limits. J. Phys. Chem. Lett. 2018, 9 (15), 4379-4384. https://doi.org/10.1021/acs.jpclett.8b01944.

(44) Zhao, Y.; Chen, F.; Li, Q.; Wang, L.; Fan, C. Isothermal Amplification of Nucleic Acids. Chem. Rev. 2015, 115 (22), 12491-12545. https://doi.org/10.1021/acs.chemrev.5b00428.

(45) Liu, H.; Li, L.; Duan, L.; Wang, X.; Xie, Y.; Tong, L.; Wang, Q.; Tang, B. High Specific and Ultrasensitive Isothermal Detection of MicroRNA by Padlock Probe-Based Exponential Rolling Circle Amplification. Anal. Chem. 2013, 85 (16), 7941-7947. https://doi.org/10.1021/ac401715k.

(46) Jiang, H.-X.; Liang, Z.-Z.; Ma, Y.-H.; Kong, D.-M.; Hong, Z.-Y. G-Quadruplex Fluorescent Probe-Mediated Real-Time Rolling Circle Amplification Strategy for Highly Sensitive MicroRNA Detection. Anal. Chim. Acta 2016, 943, 114-122. https://doi.org/10.1016/j.aca.2016.09.019.

(47) Hu, J.; Liu, M.-H.; Zhang, C.-Y. Integration of Isothermal Amplification with Quantum Dot-Based Fluorescence Resonance Energy Transfer for Simultaneous Detection of Multiple MicroRNAs. Chem. Sci. 2018, 9 (18), 4258-4267. https://doi.org/10.1039/c8sc00832a.

(48) Guo, J.; Mingoes, C.; Qiu, X.; Hildebrandt, N. Simple, Amplified, and Multiplexed Detection of MicroRNAs Using Time-Gated FRET and Hybridization Chain Reaction. Anal. Chem. 2019, 91 (4), 3101-3109. https://doi.org/10.1021/acs.analchem.8b05600.

\section{TOC Graphic}


This is the initially submitted version of the paper "J. Xu, X. Qiu, and N. Hildebrandt. When Nanoworlds Collide: Implementing DNA Amplification, Nanoparticles, Molecules, and FRET into a Single MicroRNA Biosensor. Nano Letters 2021, 21(11), 4802-4808." https://doi.org/10.1021/acs.nanolett.1c01351.

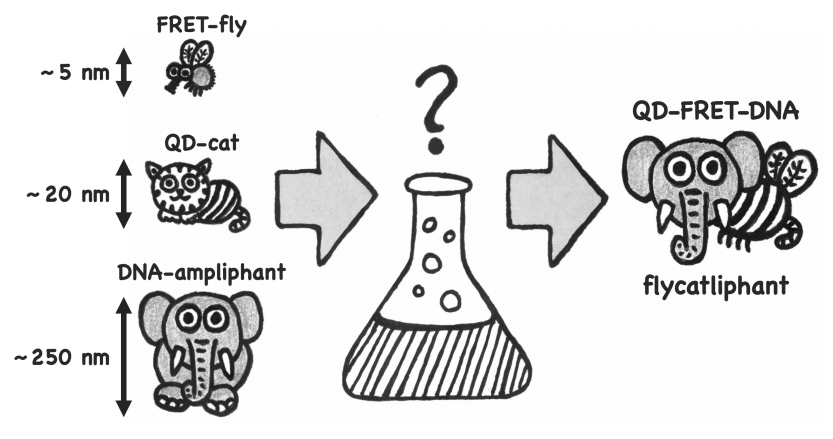

